\title{
Gradient-induced mechanical vibration of neural interfaces during MRI
}

\author{
Erwin Fuhrer, Mazin Jouda, Christopher O. Klein, Manfred Wilhelm, Jan G. Korvink (Member)
}

\begin{abstract}
Objective: Resonant vibrations of implanted structures during an MRI procedure pose a risk to the patient in the form of soft tissue irritation and degradation of the implant. In this study, the mechanical behavior of implant structures in air, water, and viscoelastic materials was explored. Methods: The static and dynamic transfer functions of various test samples in air, and immersed in both water, and hydrogels, were analyzed. The laser-based acquisition method facilitates high angular resolution $(10 \mu \mathrm{Deg})$ and high dynamic range (0 to $6 \mathrm{kHz}$ ) measurements. Additional MRI experiments were conducted to investigate the dependence of vibration strength on MR sequence parameters in combination with the obtained transfer functions. Results: The largest forces were found to be in the $\mu \mathrm{N}$ to $\mathrm{mN}$ range, which is comparable to forces applied during implantation. Of additional concern was the damping introduced by viscoelastic tissue, which was less than expected, leading to an underdamped system. In contrast to current wisdom, the imaging experiments demonstrated drastically different vibration amplitudes for identical gradient slope but different timing parameters TR, mainly due to resonant amplification. Conclusion: The results showed that a safe, force-free MR procedure depends not only on the gradient slope, but also and more drastically on the choice of secure timing parameters. Significance: These findings delineate design improvements to achieve longevity of implants, and will lead to increased patient safety during MRI. A prudent choice of mechanical characteristics of implanted structures is sufficient to avoid resonant excitation due to mismatched MR sequence parameters.
\end{abstract}

Index Terms-MRI safety, Active implants, Gradient-induced vibration, Micromotions, Transfer function

\section{INTRODUCTION}

M AGNETIC Resonance Imaging (MRI) has become one of the most important image acquisition techniques for soft tissues due to its excellent contrast ratio and noninvasiveness. In the daily clinical routine, MRI is applied to obtain anatomical cross section images or 3D volume datasets of a vast range of body parts such as joints, blood vessels, abdominal and pelvis organs and, most importantly, the central

Manuscript received October, 30, 2018; revised March 21, 2019; accepted June 07, 2019.

EF, MJ and JGK would like to acknowledge support from the BrainLinksBrainTools Cluster of Excellence funded by the German Research Foundation (DFG, grant number EXC 1086), an Advanced Grant from the European Research Council (ERC 290586 NMCEL), and the European Union's Future and Emerging Technologies Framework (H2020-FETOPEN-1-2016-2017-737043TISuMR).

Erwin Fuhrer, Mazin Jouda and Jan G. Korvink are with the Institute of Microstructure Technology, Karlsruhe Institute of Technology, EggensteinLeopoldshafen, BW, 76344, Germany (Corresponding authors: Erwin Fuhrer and Jan G. Korvink) e-mail: (fuhrerwin@gmx.de; jan.korvink@kit.edu).

Christopher O. Klein and Manfred Wilhelm are with the Institute for Technical Chemistry and Polymer Chemistry, Karlsruhe Institute of Technology, Karlsruhe, BW, 76128 Germany nervous system. Generally, MRI is considered a safe procedure because of the absence of ionising radiation. For patients carrying implants, however, MRI has been mostly contraindicated based on hazards that originate from the interaction of the electromagnetic fields of the MR unit and the conductive and/or magnetic structures of the implant. Importantly, these patients would benefit most from frequent MR scans, to give but one example, postoperative examinations. Hence, the development of MR compatible implants is imperative to enable clinicians and radiologists to conduct safe MRI based diagnostics of implant recipients [1]. Research, focused on studying interactions between MRI environment and implants, has yielded technical specifications and standards which define the proper labelling of evaluated implants [2] and provide protocols to assess heating [3], [4], image artifacts [3], [5], extrinsic electric potential [3], and induced forces in implants [3], [6], [7]. The group of forces can be subdivided in translational forces [6] and torque [7]. Considerable translational forces occur when magnetizable materials are located in the static magnetic fringe field at the bore entrance [8]. Non-magnetic materials are required to inhibit the manifestation of these interactions. Torque is generated when an object possesses an unaligned magnetic moment and is exposed to a magnetic field. Objects exhibit magnetic moments because of magnetism of matter and/or due to eddy currents induced by time varying magnetic fields in conductive materials. There are two sources for eddy currents during an MRI procedure: I. Crossing the fringe field during patient transport into the MR scanner. II. The rapid switching of the gradient fields which are required for the spatial encoding of the MR image. Avoiding magnetic materials is mostly feasible while the renunciation of conductive structures is impossible because of functional reasons, for example, in active stimulation implants that are based on electrical signaling.

The torque that manifests during patient transport may be considered static, because transportation is a slow and onetime process with a velocity about a tenth of a meter per second [9]. In contrast, induced torque based on gradient switching must be considered a dynamic process, because MR imaging is based on rapid and recurring pulses of gradient switching covering a bandwidth up to the double-digit $\mathrm{kHz}$ range [10]. Unfortunately, this range overlaps exactly with the mechanical resonance range of implanted structures and the skull [11]. Consequentially, we have to assume that there is a certain risk to excite vibrations on resonance which leads to large amplitudes. Each MR sequence has a different frequency composition, and without the knowledge of the mechanical transfer function of implants inserted in tissue, 


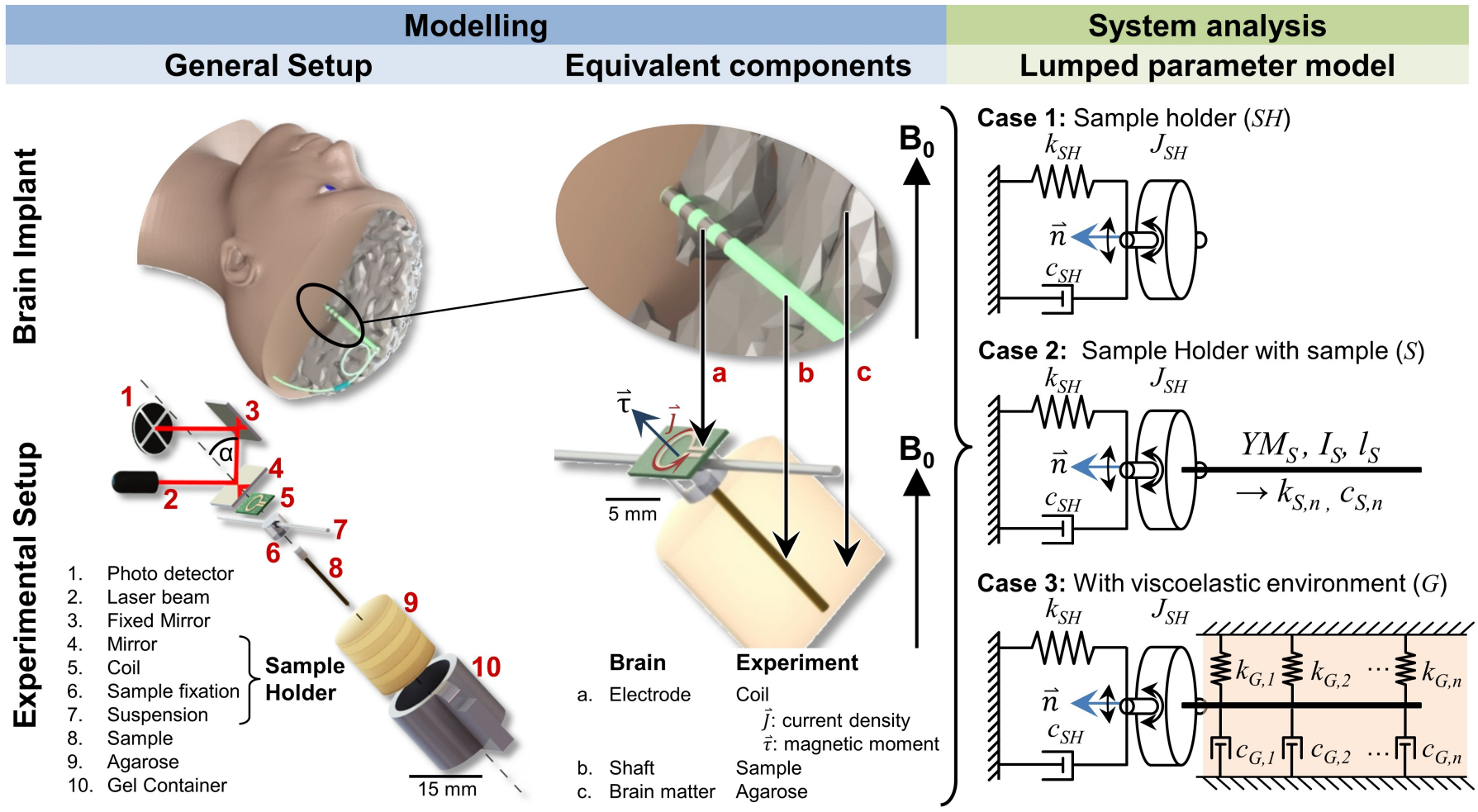

Figure 1: From model building to system analysis. Top left: Location of an electrode inside the brain. Bottom left: Schematic representation of the measurement setup. Vibration of needle structures cause a deflection of reflected Laser beam which is precisely measured using a segmented photodiode. Middle: Structures of a penetrative implant and equivalent functional structures in the measurement setup. The coil represents conductive structures and is driven externally to produce a torque with defined frequency and magnitude. Alternatively, the coil is short-circuited and currents induced by gradient switching during an MR sequence lead to time dependent torque. The shaft is represented by test beams and the brain environment is modelled with agarose. Right: Lumped parameter model which represents the measurement setup and the implanted structure. Case 1: Calibration measurements to evaluate the parameters $k_{S H}, c_{S H}$ and $J_{S H}$. Case 2: Experiments to determine the eigenfrequency of the first harmonic of the beam and its intrinsic damping behavior. Case 3: Final experiments to investigate the modified beam vibration within the viscoelastic environment to evaluate the influence of the additional spring and damping load.

it is impossible to assess and minimize the risk of resonant excitation. Only few studies, however, were conducted that investigate the effect of torque in MRI originating from eddy currents in implanted structures. Condon et al. [12] computed the amplitude of the static torsional moment of metallic heart valves based on induction. This study revealed that the torque can be significant enough, to distort the intended functionality of the valves by reducing the cardiac output. Graf et al. [13] investigated the magnitude of torque acting on different metallic frames induced by movement $(0.2 \mathrm{~m} / \mathrm{s})$ through the fringe field and during gradient switching by measuring the deflection. In the latter experiments, gradient switching revealed no measurable rotation for all samples but a perceptible vibration when touched by hand, hence, quantitative data of the torque vibration could not be extracted. Two more reports detail the torsional moments acting on heart valve prostheses. Golestanirad et al. [14] concluded that the effect of induced vibration through gradient switching can be neglected because of the small amplitudes of the gradient fields. Edwards et al. [15] built a flow rate setup to investigate potential flow rate irregularities of heart valves. These experiments revealed flow irregularities when valves were moved in the fringe field. No experiments were reported which examined the impact of gradient switching, but the au- thors highlighted that vibration induced by gradient switching may impede functional performance. Indeed, strong vibrations are not only of pure theoretical nature as shown by a case reported by Shellock [16]. This study presented a patient who reported the feeling of heat sensation, which in retrospect, was found to be caused by vibration during a certain MR pulse sequence. Considering the high demands for the functional longevity of active brain implants, knowledge of gradientinduced vibrations is of utmost importance. Several non-MRI related studies demonstrated that the micromotion between an implant and its tissue environment is an important and crucial parameter affecting the duration of functionality [17]-[20]. In the study of Sridharan et al. [21], forces acting on microelectrodes (stainless-steel, $200 \mu \mathrm{m}$ diameter) during implantation are reported to have a range of up to $1 \mathrm{mN}$. A comprehensive review by Prodanov et al. [22] captures the current understanding of mechanical interactions of an implant with brain tissue, and the impact of micromotion with respect to tissue reaction, and concludes that many more experiments considering mechanical interactions are required. Different implant fixation configurations are also presented in that review. Considering the sparse empirical data, it is imperative to quantify the gradient-induced vibration of implanted structures within representative viscoelastic environments. A very important, 
yet completely unexplored area, is the frequency-dependent transfer function of the deflection magnitude of an implant within such a viscoelastic environment. This includes the lack of data regarding the resonance conditions of implanted structures within environments such as brain matter. Only through knowledge of the damping and the spring load of the implanted electromechanical system within tissue, the resonance frequencies can be determined a priori. This data, in turn, enables the prevention of adverse amplified excitation due to resonance conditions.

This study was designed to evaluate the potential risk of amplified resonant vibration of penetrating brain implants during MRI acquisition procedures. Therefore, we investigated the mechanical behavior of electromechanical test structures within viscoelastic environments (agarose gels). These measurements yield important quantitative data for deflection amplitudes in static and dynamic states. We also measured the deflection amplitudes during different MR acquisition sequences. The quantitative data acquired during common MRI procedures allowed the additional comparison with forces occurring during an implantation procedure [21]. Hence, potential forces and risks originating from MR-induced vibrations can be analyzed in the context of established nonMRI studies. Integration of the entire measurement setup in a $1 \mathrm{~T}$ small animal MRI system enabled the characterisation of deflection amplitudes while running different imaging sequences composed of multiple frequency components, which allowed us to evaluate the induced torque caused by gradient switching. A specially constructed magnetic resonance probe, including an optical detection unit, is used for the presented experiments. The details of the probe have been first reported elsewhere [23].

\section{Methodology And System Design}

\section{A. Principle of measurement method and system setup}

The experimental setup is designed to emulate active penetrating implants within tissue to evaluate mechanical implanttissue interaction. Fig. 1 displays and indicates the functional parts of a real implant and the equivalent structures in the developed experimental setup. The main structural feature of all penetrating implants is the shaft in which electrodes and electrical connections are embedded. Most shafts are based on polymers, silicon, or ceramic materials [22], [24]. Implant shafts, from micro-structured arrays to macroscopic implants such as implants for deep brain stimulation (DBS) (illustrated in Fig. 1), have high aspect ratios which enable deep tissue penetration while avoiding extensive tissue displacement or rupture. To mimic these shafts, we used $20 \mathrm{~mm}$ long cylindrical rods as test samples, varying in material and diameter. We used copper $(\mathrm{Cu})$ and brass $(\mathrm{Br})$, which represent crystalline materials (high Young's Modulus and low damping factor) and acrylonitrile-butadiene-styrene (ABS) representing an amorphous polymer material (low Young's Modulus, high damping factor and low glass transition). In the following text these rods are referred to as "sample". All penetrating implants are mechanical structures embedded within the brain tissue, which is a viscoelastic environment. A standard approach to mimic the brain environment is the use of agarose gels [25]. Prepared with different concentrations, agarose gels yield variations in mechanical properties, and most importantly, an increase of shear modulus for both the storage $G^{\prime}$ and loss modulus $G^{\prime \prime}$ with ascending concentration (small-strain and low frequency regime). Hence, gels with different concentration can be used to emulate different viscoelastic brain tissues. Probably the most important structural feature of an active penetrating implant are the electrodes arranged on the shaft for signaling or stimulation. Gradientinduced torque on the shaft is due to induced eddy currents in the electrodes. This is emulated in our setup using a coil with external current supply (Fig. 1 left and middle column). The magnitude of gradient-induced torque $T_{\text {ext }}$ may vary by many orders dependent on the shape of the conductive component, its orientation to $\mathbf{B}_{0}$, and the gradient fields, the slew rate, and the magnitude of $\mathbf{B}_{0}$ (see appendix $B$, equation 4 ). The torque can be imitated by an externally operated coil with surface $A$, which enables the precise control of the applied torque $T_{\text {ext }}$ through a defined current $i(t)$ over several orders of magnitude $\left(T_{\text {ext }}(t)=A \cdot i(t) \times \mathbf{B}_{0}\right)$. Furthermore, this approach allows the user to measure the transfer function with a frequency sweep at constant torque.

The sample holder has no corresponding part in the brain. However, it is required to integrate the coil and mirror and to provide an interface for exchangeable samples. This applied design provides the connection to the external frame using a torsion bar suspension. Three different sample holders were fabricated, and differ only in diameter and material of the two torsion bar suspensions. The sample fixation unit is integrated to support exchangeable mounting of the sample. On top of this fixation unit, the coil and the mirror are adhesively attached. The sample fixation sandwich, including unit, coil, and mirror, is mounted at an angle of $45^{\circ}$ with respect to the external $\mathrm{B}_{0}$-field. The motion of a reflected laser beam is detected using a segmented photodiode. Using an undoped silicon chip as a mirror is beneficial compared to metal-based mirrors, because of the absence of parasitic eddy currents during MRI procedures.

The presented measurements are designed, to explore the statics and dynamics arising from sample-agar interaction, and the resulting mechanical behavior of this composition during MRI. By exploiting the modularity of our setup, we measured the transfer functions of different samples which were embedded in air, water and agarose-based hydrogels of different concentrations. Of paramount interest is the difference of the transfer functions that was obtained for the same sample consecutively immersed in these various environments. Most importantly, the data provides important information with which to assess the risk of amplitude resonances during an MR session.

\section{B. Experimental control, data processing and analysis}

The scheme for experimental control and signal flow is displayed in Fig. 2. The coil acts as a current-torque transducer if placed inside a magnetic field, and enables the controlling of torque by adjusting the voltage using the frequency generator 


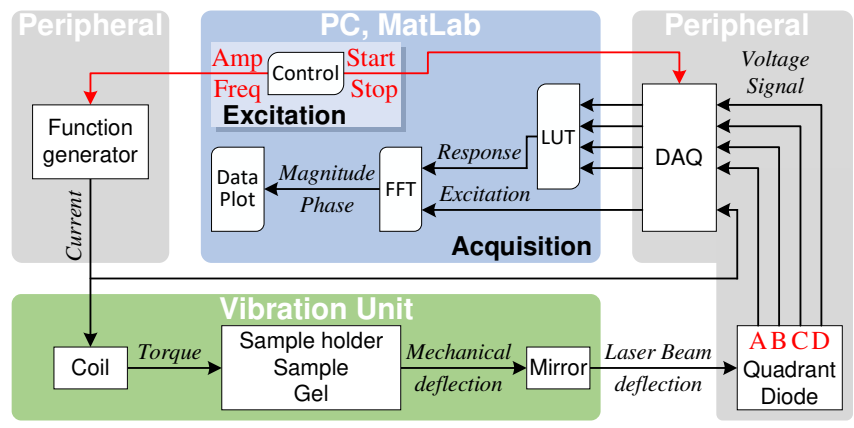

Figure 2: Flowchart of the MatLab controlled excitation and acquisition scheme. A function generator is used in combination with a variable resistor to produce a peak current in the range of $4 \mu \mathrm{A}$ to $0.4 \mathrm{~A}$. The current signal was recorded. The induced vibration was measured by digitizing the analogue output signal from a segmented diode, and subsequent conversion to a deflection angle using a calibrated Lookup-Table (LUT).

Tektronix AFG-3022B in combination with a series resistor board. The deflection was measured by converting the voltage signals of the segmented diode into $x-y$-position data using a look-up-table which was calibrated on an optical bench. Data collection was realized using the digital acquisition modules RedLAB 1408-FS (Meilhaus) for the excitation signals and NI-9215 (National Instruments) for the segmented diode signals using oversampling[26]. To enable synchronization, one diode signal was split and fed into both acquisition devices. Controlling and acquisition was realized through interfacing via MatLab (Version 2018a). Post-processing, such as voltageto-position conversion, FFT-based analysis, and data visualization, was conducted using MatLab and InkScape.

\section{Materials and characterization methods}

In the following, materials used for sample holder, samples, gels, and the required characterization methods, are described. An overview is provided by table I.

Sample holder: The various sample holders differ only in their suspension (see Fig. 1 label 7). We used suspension rods made from PMMA, copper $(\mathrm{Cu})$, and nylon $(\mathrm{Ny})$, with a variation in diameter to obtain different stiffnesses.

Samples: Samples were tested with respect to their change of transfer function within different environments. All samples have a length of $20 \mathrm{~mm}$. Materials used for the samples were copper $(\mathrm{Cu})$, brass $(\mathrm{Br})$ and $\mathrm{ABS}$ with diameters of $0.25 \mathrm{~mm}$, $0.5 \mathrm{~mm}$ and $2 \mathrm{~mm}$. The sample with mass is a $0.25 \mathrm{Cu}$ sample equipped with a $140 \mathrm{mg}$ weight (drop-like form, largest diameter: $2.5 \mathrm{~mm}$ ) at the tip to reduce the resonance frequency of the beam.

Agarose gels: We measured the transfer function of the samples within air, water, and agarose gels having ascending concentrations. The agarose gels were prepared using four different weight concentrations $(0.5 \mathrm{~g}, 1 \mathrm{~g}, 2 \mathrm{~g}, 4 \mathrm{~g})$ of agar powder (AGAR AGAR SERVA 11396.02) dissolved in $100 \mathrm{ml}$ de-ionized water, and an additional 13-vol \% of N-propanol according to the protocol presented by Kavitha et al. [27]. These values were chosen to approximate the mechanical properties of the brain (see Ref. [21], [28], [29]). Here,
Table I: Properties and materials of sample holder, test samples, and test environments. This modular setup lead to a total number of 144 combinations of sub-units (sample holder + sample + environment). Additionally, the sample holders were characterized without load. The measurements furthermore were separated in static and dynamic measurements, leading to a total amount of 296 possible combinations.

\begin{tabular}{|c|c|c|c|c|c|}
\hline \multicolumn{2}{|c|}{ Sample holder } & \multicolumn{2}{|c|}{ Test sample } & \multicolumn{2}{|c|}{ Test environment } \\
\hline$\emptyset[\mathrm{mm}]$ & Material & $\varnothing[\mathrm{mm}]$ & Material & Concentration & Material \\
\hline 0.25 & $\mathrm{Cu}$ & 0.25 & $\mathrm{Cu}$ & - & Air \\
\hline 0.5 & $\mathrm{Cu}$ & 0.25 & $\mathrm{Cu}+$ mass & - & Water \\
\hline 0.5 & $\mathrm{Ny}$ & 0.5 & $\mathrm{Cu}$ & $0.5 \%$ & Agarose \\
\hline 2 & PMMA & 0.5 & $\mathrm{Br}$ & $1 \%$ & Agarose \\
\hline & & 0.5 & ABS & $2 \%$ & Agarose \\
\hline & & 2 & $\mathrm{Br}$ & $4 \%$ & Agarose \\
\hline
\end{tabular}

the $0.5 \%$ gel resembles the healthy brain's tissue, with a shear modulus of approximately $2 \mathrm{kPa}$. Higher concentrated gels emulate post-surgical tissue reaction, which leads to temporal increase of shear modulus up to $45 \mathrm{kPa}$ [22] and which corresponds to a $4 \%$ gel. Special gel geometries (discs with $40 \mathrm{~mm}$ diameter and $10 \mathrm{~mm}$ height) were made for the characterization using the rheometer ARES-G2 from TA instruments. For the vibration experiments inside the MRI, small gel containers were filled with warm, liquid agarose gel, followed by a one hour of cooling. The vibration experiments were conducted using the custom-made probe, designed for a $1 \mathrm{~T}$ small animal MRI system (ICON, Bruker).

\section{System analysis and experiments}

To analyze the underlying mechanics of the experimental setup, we start with a two degree-of-freedom (DOF) lumped parameter model. This model allows the assessment of the dynamics between sample holder, sample, and gel, requiring a reduced number of required experiments by choosing correct component configurations, and enabling analysis of the obtained data. The moment of inertia of the sample holder is represented by the mass element $J_{S H}$. The torsion bar suspension of the sample holder is represented by the springdamper system $\left(k_{S H}, c_{S H}\right)$ connected to this mass and the ground (the frame). Different samples, all cantilever structures, mounted to the sample holder, are represented by a springdamping system $\left(k_{S}, c_{S}\right)$ with a mass $J_{S}$. Finally, the agar gel is represented by a spring-damper system $\left(k_{G}, c_{G}\right)$ that connects the mass of the sample to ground (the frame). In Fig. 3 the corresponding lumped model is visualized.

We are interested in the motion of $\alpha_{2}(t)$ which represents the beam motion. Since the deflection signal measures $\alpha_{1}(t)$ of the sample holder, the deflection $\alpha_{2}(t)$ is measured indirectly using the mechanical coupling to $\alpha_{1}(t)$. The coil is mounted onto the sample holder, hence, the external force acts on the mass $m_{S H}$. Indirect measurement analysis is enabled through the modularity of the experimental setup, which allows measuring a sample holder without sample (see Fig. 1 case $1 ; c_{S}, k_{S}, m_{S}, c_{G}, k_{G}=0$ ), with the sample (case $2 ; c_{G}, k_{G}=0$ ), and subsequently to conduct a measurement within different viscoelastic environments (case 3). Furthermore, we can separately conduct dynamic and static measurements. In the static case the lumped model 


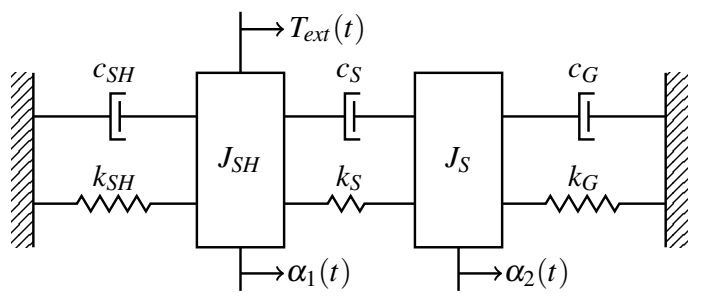

Figure 3: Reduced lumped parameter model, including the moment of inertia, springs, and damping of the sample holder $(\mathrm{SH})$ and the sample (S) and agarose gel $(\mathrm{G})$. The spring constant $k_{G}$ correlates with the storage modulus $G^{\prime}$ and the damping constant $c_{G}$ correlates with the loss modulus $G^{\prime \prime}$ of the gel. External torque $T_{\text {ext }}$ can only be applied to the sample holder which the coil is attached to. Since the mirror is attached to the sample holder too, we can only measure the deflection of $\alpha_{1}(t)$, from which we infer $\alpha_{2}(t)$.

simplifies to a plain spring network which can then be characterized, because terms with time derivatives vanish. In this case, the total spring constant $k_{t o t}$ consists of the spring of the sample holder $k_{S H}$ which lies parallel to the serial-connected springs of the sample $k_{S}$ and the gel $k_{G}$. The total spring constant can be obtained by applying an external torque $T_{\text {ext }}$ and then measuring angular deflection $\alpha_{1}$.

1) Dynamic measurements: The dynamic behavior of the system is modelled by the following $2^{\text {nd }}$-order differential equation with the deflection vector $\alpha(t)=\left(\alpha_{1}(t) \alpha_{2}(t)\right)^{T}$ :

$$
\mathbf{J} \ddot{\alpha}+\mathbf{C} \dot{\alpha}+\mathbf{K} \alpha=\mathbf{B} T(t)
$$

$\mathbf{J}, \mathbf{C}, \mathbf{K}$ are matrices representing the moment of inertia, damping and spring, respectively (for matrix elements see appendix A). Externally applied torque is given by $\mathbf{B}=\left(\begin{array}{ll}1 & 0\end{array}\right)^{T}$, so that no external force is directly acting on the mass of the sample.

2) Static measurements: In the static condition the time dependent terms of equation $1 \mathbf{J} \ddot{\alpha}$ and $\mathbf{C} \dot{\alpha}$ equal zero. Hence, the ratio of the externally applied torque $T_{\text {ext }}$ to deflection $\alpha_{1}$ equals the total spring constant $k_{t o t}$ of the setup:

$$
\frac{T_{e x t}}{\alpha_{1}}=k_{S H}+\frac{k_{S} k_{G}}{k_{S}+k_{G}}=k_{\text {tot }} .
$$

In case of an empty sample holder, the spring constant of the sample and gel are $k_{S}=k_{G}=0$, hence, measuring $\alpha_{1}$ results in the spring constant of the sample holder $k_{S H}$. Subsequent addition of a sample, and measurement for all variations of gels, allows one to determine the other spring constants. The total spring constant, which depends on the gel viscosity, is valid over a wide parameter range if the following conditions are met: $k_{S H} \gg\left(k_{S}|| k_{G}\right)$ and $k_{S} \ll k_{G}$. Measurements were conducted with and without gel to determine the contribution of the individual spring components. As an example, the deflection measurements with the empty sample holder allows the determination of $k_{S H}$. All static deflection measurements were conducted at $4 \mathrm{~Hz}$. This frequency facilitates high precision digital band-pass filtering, whereas the measurements can still be considered quasi-static, as verified by gel characterization experiments.

3) MRI measurements: By utilizing the precise knowledge of the transfer function of a system we could demonstrate the effect of maximal vibration due to worst-case timing parameters of an MR sequence. We first determined the transfer function of a sample and then adjusted the MR sequence to obtain the worst-case condition. To remove the largest background noise source, we turned off the fan cooling system of the MR unit. The acoustic noise of the gradient switching was reduced by using very weak gradient fields. To compensate for the reduced magnitude of torque, we connected a larger coil (10 $\mathrm{mm}$ diameter) in parallel to the installed coil. Sufficient acoustic decoupling was tested by conducting measurements with open coil terminals resulting in no vibration. Vibration tests during MRI were performed on a $0.5 \mathrm{~mm}$ copper beam immersed in water and mounted on the $0.5 \mathrm{Cu}$ sample holder. We conducted two experiments using identical MR sequences that only differed in repetition time TR. The applied sequences were standard two dimensional (2D) gradient echo sequences (GRE) using 10 slices with $\mathrm{TR}=200 \mathrm{~ms}\left(\mathrm{TR}_{\mathrm{eff}}=20 \mathrm{~ms}\right)$ and $\mathrm{TR}=141.9 \mathrm{~ms}\left(\mathrm{TR}_{\mathrm{eff}}=14.19 \mathrm{~ms}\right)$, respectively. To ensure induction from only the slice selection gradient (10 $\mathrm{mm}$ ), we set the phase and read encoding gradients to a very large field-of-view (FOV, $1000 \mathrm{~mm}, 32 \mathrm{pixel})$. This approach allowed for precise monitoring of the driving gradient without superposition of the two other gradient fields. After these tailored vibration tests for minimal background and acoustic noise coupling, we conducted the same sequences, however, with a slice thickness of $0.5 \mathrm{~mm}$ and an FOV of $15 \mathrm{~mm}$ (128 pixel). Here, MR signal acquisition was enabled using an RF coil wrapped around the water container. Simultaneous readout on the optical channel confirmed the results of the previous vibration measurements, but additional background noise was observed.

\section{RESUlTS AND DISCUSSION}

\section{A. Simulation \& Modelling}

Theoretical results of static deflection were obtained using the lumped model and displayed in Fig. 4. These graphs show the deflection as a function of the spring constant $k_{G}$ for normalized torque (arbitrary units), hence, the graph ordinate is equivalent to the inverse of the total spring constant $k_{t o t}$. For small values of $k_{G}$, the deflection is dominated by $k_{S H}$ (blue encoded area), whereas for very large values of $k_{G}$ the deflection is dominated by $k_{S}$ (grey encoded area).

Analytical results of the beam dynamics are shown in Fig. 5 for: a) the magnitude for $\alpha_{1}(f)$ (directly measurable signal) and b) the magnitude for $\alpha_{2}(f)$ (non-measurable). The transfer function was obtained by Laplace transformation of equation 1. The transfer function of the sample without gel $\left(k_{G}=0\right)$ is visualized by the blue lines. A clear amplitude resonance is observable from the blue peaks. An additional spring constant with no damping leads to a shift upwards in frequency (red) and a decrease in magnitude. In addition to the spring load, the gel introduces damping through which the peak observed at coordinate $\alpha_{1}$ may become very small in 


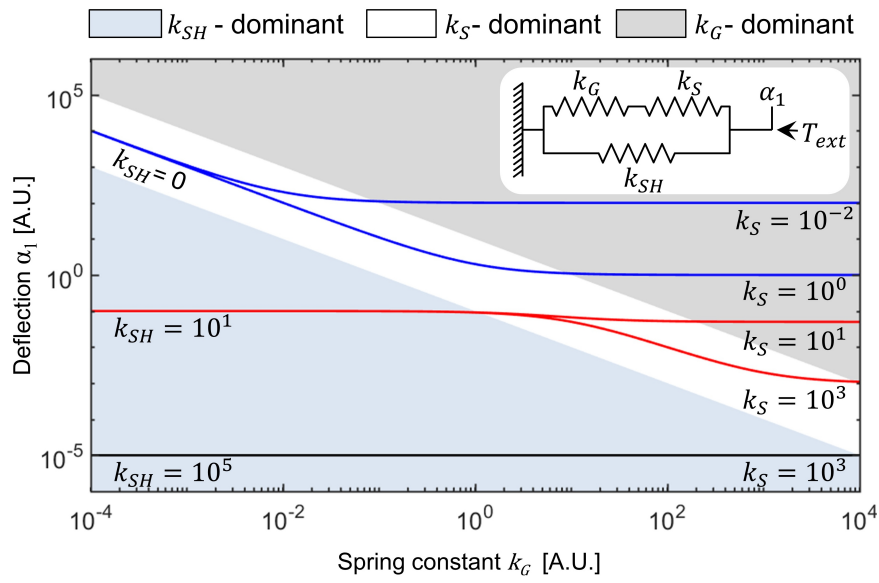

Figure 4: Computed deflection of the total spring system as a function of spring constant $k_{G}$ per unit torque. The color encoded regions indicate where the deflection (or inverse of total spring constant) depends primarily on one spring only. The model in the inset is obtained by removing the time dependent elements of Fig. 3

amplitude and decreases in frequency. If no resonance peak is detected we may conclude that the reason is strong damping or even overdamping, however, this conclusion may be a fallacy. Instead, at this point we can only deduce that the maximum amplitude at resonance is simply below the display resolution limit. This can be seen from the inset of Fig. 5, where the weak resonance of the amplitude peak of the sample leads only to a slight increase of magnitude on the sample holder, and which is much weaker compared to the other two peaks. Additionally, this analysis shows the advantage of selecting a sample holder with a resonance frequency $f_{\text {Res }, S H}$ larger than the resonance frequency $f_{\text {Res }, S}$ of the sample, simply because above $f_{\text {Res }, S H}$ the signal decays with $20 \mathrm{~dB} /$ decade due to its low pass behavior. This approach thereby ensures maximal signal-to-noise-ratio (SNR) to measure the sample resonance.

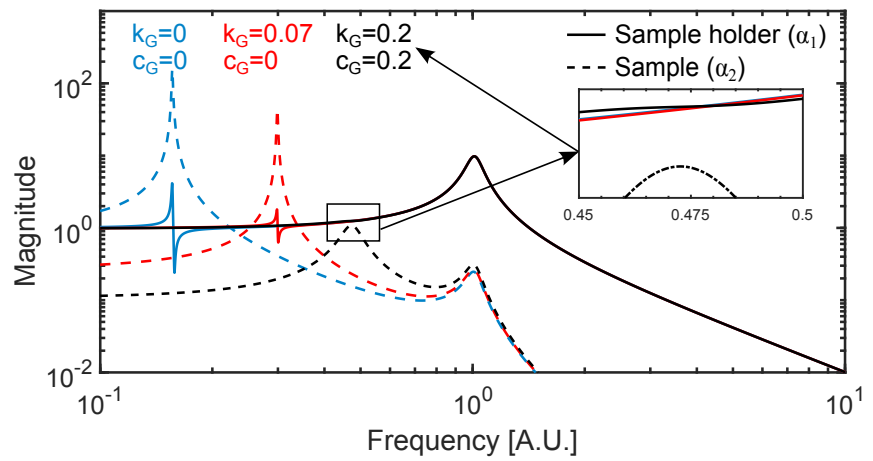

Figure 5: Computed transfer functions based on equation 1 for different spring constant $k_{G}$ and damping constants $c_{G}$. Other values are: moment of inertia: $J_{S H}=J_{S}=1 ;$; springs: $k_{S H}=1, k_{S}=0.025$ and damping constants: $c_{S H}=0.1, c_{S}=0.001$.

In case the sample peak vanishes for measurements conducted within gels, we further analyzed whether a possibility exists to extract information using the peak of $f_{R e s, S H}$. This analysis is visualized in Fig. 6. A typical magnitude plot (A) shows peaks from sample resonance (peak one indicated by an orange background) and sample holder res-
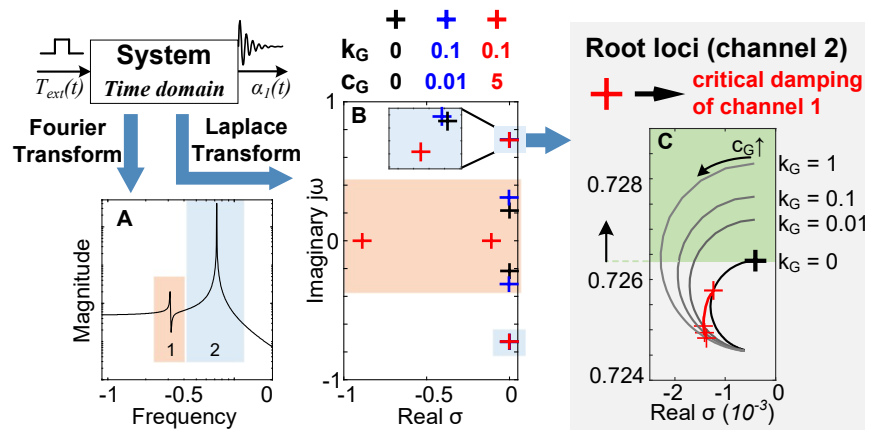

Figure 6: System analysis using Fourier and Laplace transforms. A: Magnitude plot displays the two resonances $f_{\text {Res.SH }}$ (peak 1) and $f_{\text {Res }, S}$ (peak 2) of the 2 DOF system. B: Location of poles of peak one and peak two for different gel combinations $k_{G}$ and $c_{G}$. The insert reveals the region around peak 2. C: Root loci of peak 2 for increasing damping $c_{G}$ and various spring constants $k_{G}$ of the gel. The poles of peak 2 at critical damping of the sample are marked red. The green area marks the region of frequency shift upwards of peak two.

onance (peak two indicated by a blue background). Alternatively, these resonances can be displayed in the s-Plane as poles, which is shown in Fig. 6 for three different configurations of $k_{G}$ and $c_{G}(\mathbf{B})$. For one configuration, marked in red, overdamped resonance is obtained leading to real poles (on the imaginary axis). Under this condition, peak one in the magnitude plot disappears, however, we can measure the effect of the sample-gel interaction indirectly on channel 2 (resonance peak of sample holder). This is shown in the zoomed blue square $(\mathbf{B})$ in which the separation of the poles becomes visible. Overdamping of the sample leads to a shift downwards of amplitude resonance $f_{R e s, S H}$, indicated by the red marker that has a smaller value for $j \omega$ than the black marker. Finally, the root loci for all poles of channel 2 for a variation of $c_{G}$ and $k_{G}$ are analyzed and a selection of graphs is plotted. The red line indicates the critical damping. The black marker represents the pole for the amplitude resonance in air $\left(k_{G}=c_{G}=0\right)$. An extremely important result for gel measurements is the fact that, if the resonance frequency $f_{\text {Res }, S H}$ of the sample holder increases (green surface), the sample itself resonates in an underdamped mode inside the gel. Overdamped resonance of the sample always leads to a decrease of resonance frequency $f_{R e s, S H}$, a consequence due to the fact that the red line is strictly separated from the green area.

\section{B. Gel characterization}

Results of the strain sweep oscillation experiment are shown on the left graph of Fig. 7. Up to approximately $0.1 \%$ strain the shear moduli of all the gels are constant. Deflections within this strain limit allow for linear approximation of spring behavior. Frequency shear oscillation tests conducted at $0.01 \%$ strain rate are shown on the right graph of Fig. 7. The shear moduli (real and complex parts) of all the gels are constant up to approximately $5 \mathrm{~Hz}$, which justifies to perform quasistatic measurements at $4 \mathrm{~Hz}$ where $\tan \delta=G^{\prime \prime} / G^{\prime} \rightarrow 0$. A further increase of frequency leads to an increase of the loss 


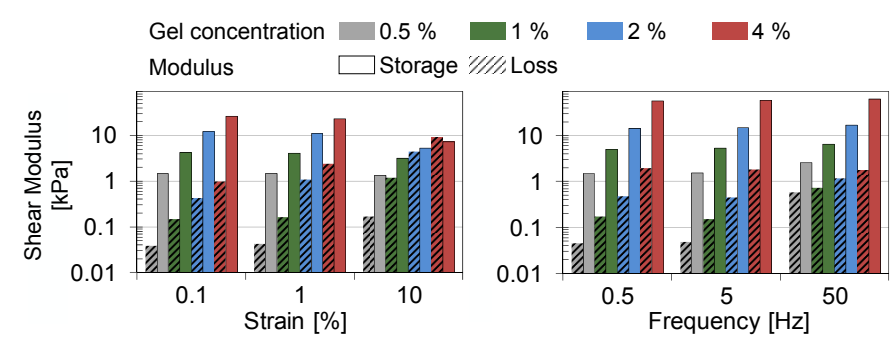

Figure 7: Left: Shear modulus as a result of stress-strain measurements, conducted at $1 \mathrm{~Hz}$. For small shear strains $(\gamma<0.1 \%)$ elasticity (storage modulus) is dominant over viscosity (loss modulus) for all gels by more than an order of magnitude. Right: Shear modulus as a result of frequency measurements measured at $0.01 \%$ strain rate. Up to $5 \mathrm{~Hz}$ the shear modulus is constant.

moduli $G^{\prime \prime}$, while the storage moduli $G^{\prime}$ are constant. This behavior, however, also depends on the agar concentration and for higher concentrations the effect becomes less pronounced and for the $4 \%$ gel the loss modulus decreases with increasing frequency. Tests conducted with larger strain rates showed more viscous behavior with increasing frequency.

\section{Transfer functions}

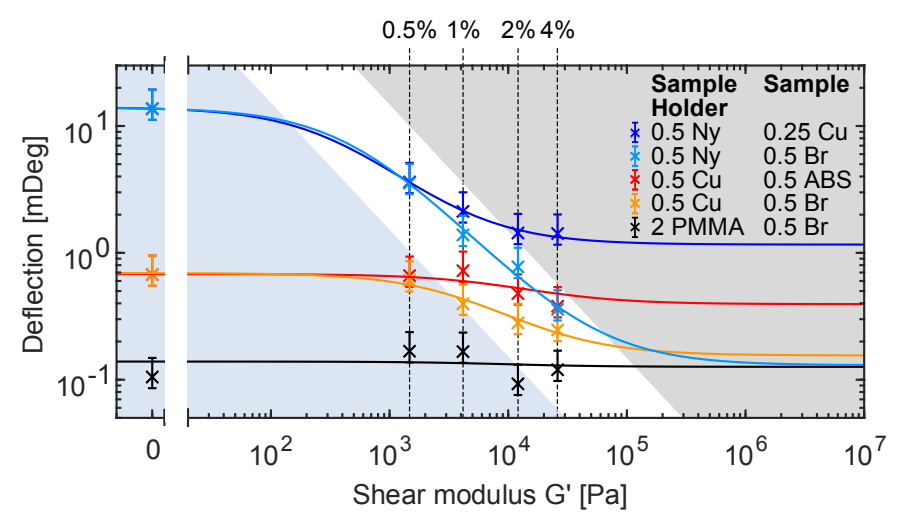

Figure 8: Results of static deflection measurements including data fits based on the lumped parameter model. The applied torque for these measurements was in the range of 0.005 to $5 \mu \mathrm{Nm}$, the displayed results are normed to a torque of $1 \mu \mathrm{Nm}$.

1) Static measurements: The most important experimental results from the static measurements are compiled in Fig. 8. Three different sample holders were employed in combination with four different samples $(2 \mathrm{Br}, 0.5 \mathrm{Br}, 0.5 \mathrm{ABS}, 0.25 \mathrm{Cu})$. The $0.25 \mathrm{Cu}$ and $0.5 \mathrm{ABS}$ resulted in similar deflection, hence only $0.25 \mathrm{Cu}$ is presented to gain greater clarity in the figures. Each measurement point in Fig. 8 is obtained by linear fitting of the data points obtained from a torque-deflection measurement. We observe strong resemblance between the computed results using the lumped model displayed in Fig. 4 and the obtained measurement results. Based on these characterization graphs we can evaluate the torque acting on the samples during an MR sequence by measurement of deflection.
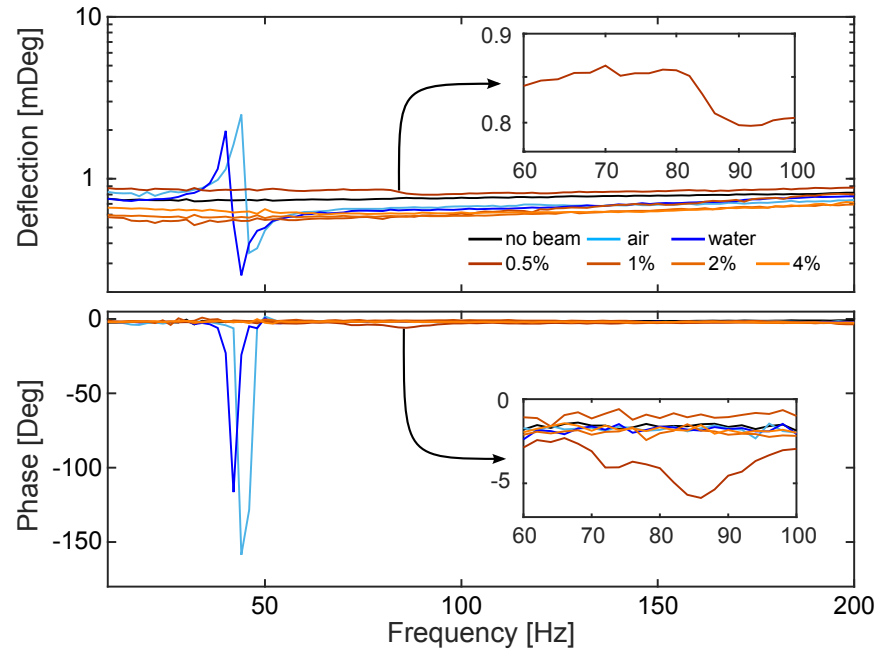

Figure 9: Complete Bode plot of the $0.5 \mathrm{Cu}+$ mass sample in combination with the $0.5 \mathrm{Cu}$ sample holder measured in air, water and all gels. Amplitude resonances in air (orange) and water (yellow) are clearly visible. The inset shows the resonance of the sample in the $0.5 \%$ gel.

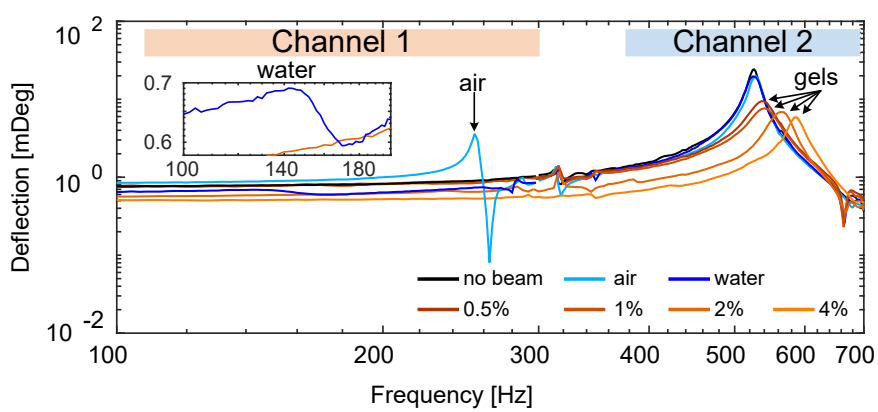

Figure 10: Transfer function of a 0.5 ABS sample in combination with a $0.5 \mathrm{Cu}$ sample holder. Resonance amplitudes within the gel are not visible, but the increase of the resonance frequency of the SH indicates the underdamped resonance condition of the sample.

2) Dynamic measurements: We measured the transfer functions for a large number of configurations of sample holder and samples. In Fig. 9, the transfer function of the sample "0.5 $\mathrm{Cu}+$ mass" mounted on the "0.5 Cu" sample holder is displayed. Amplitude resonance in air is clearly visible at approximately $45 \mathrm{~Hz}$. This resonance is slightly shifted downwards within water due to additional damping. Importantly, direct amplitude resonance is visible for the $0.5 \%$ gel with a resonance shift up to $85 \mathrm{~Hz}$ recognizable in the magnitude plot and more clearly in the phase diagram. The gel leads to a doubling of resonance frequency. Resonant frequencies for the higher concentrated gels are not visible.

Measurement results for another combination (sample: 0.5 ABS; sample holder $0.5 \mathrm{Cu}$ ) are presented in Fig. 10 . The amplitude resonance of the free beam is clearly visible at $260 \mathrm{~Hz}$. If placed inside water, this beam is strongly damped leading to a weak resulting amplitude at $180 \mathrm{~Hz}$. Amplitude resonances within the gels are not visible, however, the peaks of the sample holder experience a strong shift upwards, depending on the gel concentration. Hence, we conclude that the sample within all gels resonates in an underdamped manner, as 


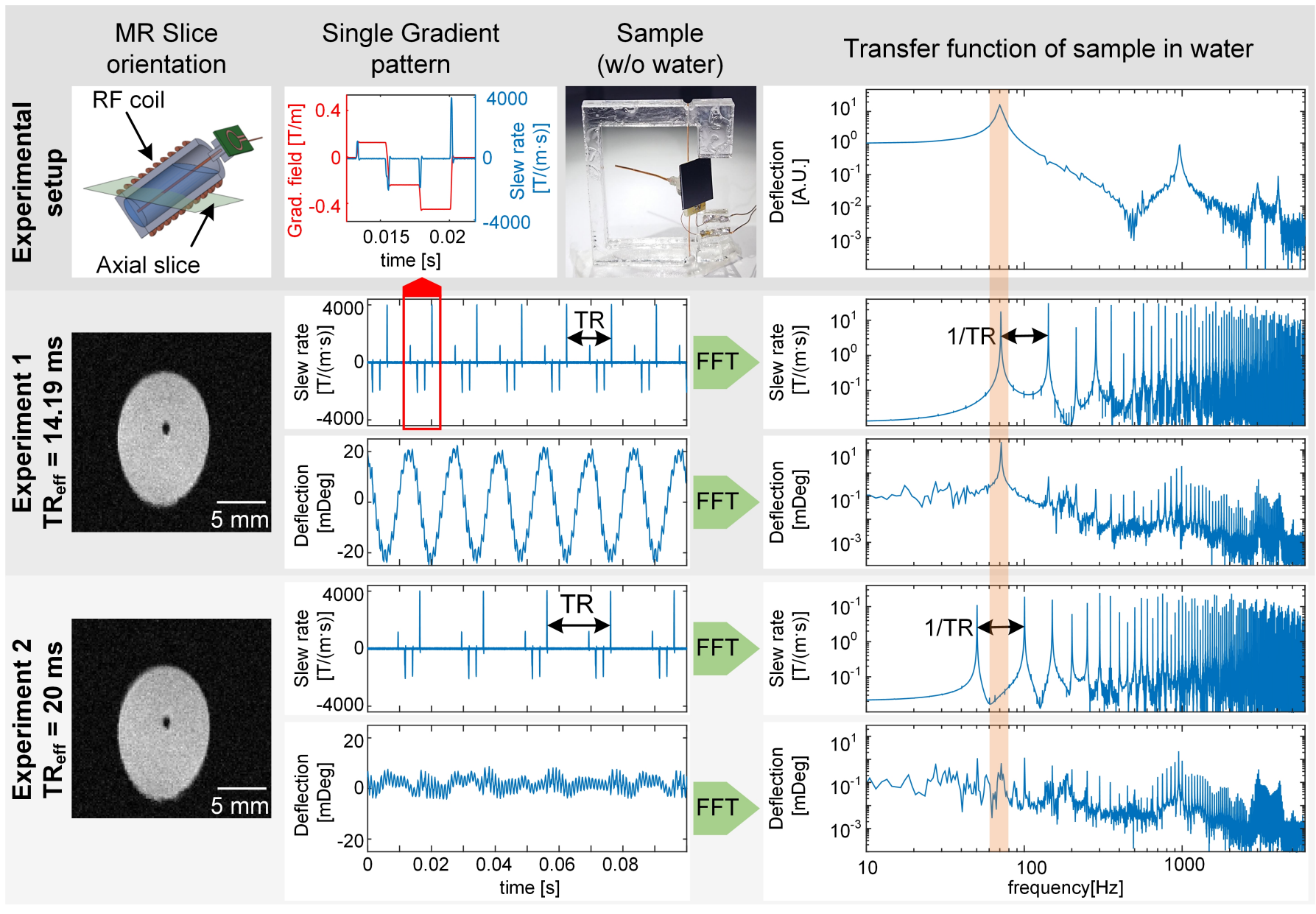

Figure 11: MR experiments demonstrating different vibration amplitudes during imaging sequences which differ only in repetition time TR. The switching from TR $=20 \mathrm{~ms}$ to $14.18 \mathrm{~ms}$ leads to a five-fold increase of vibrational amplitude. In the frequency domain, the origin of the increased vibrational amplitude is easily discovered: spectral components of the gradient switch pulse overlap with the resonance frequency of the mechanical system (area highlighted in orange).

analyzed within Fig. 6. Generally, a system is underdamped if the damping ratio $\zeta(f)=c(f) / 2 \sqrt{J k(f)}<1$. The damping ratio in arbitrary viscoelastic environments depends on their viscoelastic properties, as well as the magnitude and frequency of the vibration. Quantitative data with respect to other tissue types (e.g. muscles) require experimental validation.

\section{MRI images}

In Fig. 11 the orientation of the MR slice, the shape of one single gradient pulse in the time domain, the sample, and the transfer function, are illustrated within the panel "Experimental setup". Within the two other panels, we present both the time domain and frequency domain signal of the gradient switching, and the resulting vibration for the two MR experiments that use sequence parameters that differ only in repetition time TR. In the first experiment we set TR to match the first resonance of the previously obtained transfer function. This results in a clearly visible strong vibration amplitude of $20 \mathrm{mDeg}$ with a frequency of $70.5 \mathrm{~Hz}$ corresponding to a period of $14.19 \mathrm{~ms}$. In the second experiment we chose a repetition time that has no spectral components around the first resonance. Changing TR to $200 \mathrm{~ms}\left(\mathrm{TR}_{\mathrm{eff}}=20 \mathrm{~ms}\right)$ leads to four-fold decrease of the peak amplitude. In this case, the main vibration originates from the second resonance of the system at approximately $1 \mathrm{kHz}$. Importantly, these vibrations are not observable in the MR image (e.g. as an artefact) and thus invisible. This result demonstrates unequivocally the impact of MR sequence timing parameters on the resulting vibration amplitudes. On the other hand, we want to emphasize that largely amplified resonance amplitudes require weakly dampened mechanical environments, such as the one prepared with water. Vibration measurement in the hydrogels resulted in a larger damping behavior compared to the measurements in water, however, not to the extent that the systems were overdamped. This means that amplified vibration amplitudes may occur also in viscoelastic environments, however, with a reduced level of amplification compared to water. On the other hand, the larger the damping due to the viscoelastic materials the more energy is dissipated by the surrounding material rather than in the beam.

\section{CONCLUSION}

We presented an accurate methodology that allows the user to analyze MRI-induced vibration of penetrative implants 
theoretically and experimentally, including the probe dynamics within a brain-tissue model. The modular setup enabled the measurement of a large number of design combinations for implant-tissue models, yielding an extended dataset, which in turn facilitated the validation of simplified analytical models used for static and dynamic analysis. Furthermore, the experimental setup was integrated in an MR unit, which allowed to evaluate potentially critical MR sequences for the characterized systems. The major key finding is, that for all viscoelastic embedding materials, underdamped resonance conditions are found. This result emphasizes the potential risk of amplified vibrations due to accidental adverse on-resonant excitations. At this point, however, the question remains open as to how strong mechanical vibrations will influence an implant's functionality, and whether it will impede its lifetime. These questions concern not only the degree of degradation of the hardware, but also the tissue reaction (e.g. inflammatory response) as a function of vibrational amplitude and frequency. Both of these aspects were not investigated in the current work. Hence, more research will be required to understand the impact of resonant vibrations on probe functionality and longevity, which, in turn will allow a first attempt at risk analysis.

\section{APPENDIX A \\ MATRICES OF TRANSFER FUNCTION}

The matrices in equation 1 are the inertia matrix $\mathbf{J}$, the damping matrix $\mathbf{D}$, and the spring matrix $\mathbf{K}$ :

$$
\mathbf{J}=\left(\begin{array}{cc}
J_{1} & 0 \\
0 & J_{2}
\end{array}\right), \mathbf{C}=\left(\begin{array}{cc}
c_{1}+c_{2} & -c_{2} \\
-c_{2} & c_{2}+c_{3}
\end{array}\right), \mathbf{K}=\left(\begin{array}{cc}
k_{1}+k_{2} & -k_{2} \\
-k_{2} & k_{2}+k_{3}
\end{array}\right)
$$

\section{APPENDIX B}

\section{GRADIENT-INDUCED TORQUE}

The torque that acts on a conductive component within a static magnetic field due to gradient switching is given by the following equation [3]:

$$
T(t)=\underbrace{\frac{1}{8} \pi \sigma h r^{4}}_{\text {Implant }} \underbrace{\sin (\alpha) \cos (\beta)}_{\text {Orientation }} \underbrace{\dot{B}(t) B_{0}}_{\text {MR environment }}
$$

Here, the parameter $\sigma$ represents the conductivity, the parameters $h$ and $r$ are the height and radius, respectively. The variable $\alpha$ is the angle between the normal of the implant surface and the vector of $\mathrm{B}_{0}$, and $\beta$ is the angle between the normal and the gradient field vector. $\dot{B}(t)$ represents the time dependent slew rate of the gradient field, and $B_{0}$ is the magnitude of the static field.

\section{REFERENCES}

[1] J. B. Erhardt et al., "Should patients with brain implants undergo MRI?" Journal of Neural Engineering, vol. 15, no. 4, p. 041002, aug 2018.

[2] "ASTM F2503 - 13: Standard Practice for Marking Medical Devices and Other Items for Safety in the Magnetic Resonance Environment 1," pp. 1-7, 2013.

[3] International Organization for Standardization, "ISO/TS 10974:2012 Assessment of the safety of magnetic resonance imaging for patients with an active implantable medical device," p. 200, 2012.
[4] "ASTM F2182 - 11a: Standard test method for measurement of radio frequency induced heating near passive implants during magnetic resonance imaging," pp. 1-14, 2011.

[5] "ASTM F2119 - 07: Standard Test Method for Evaluation of MR Image Artifacts from Passive Implants 1," pp. 1-4, 2013.

[6] "ASTM F2052 - 15: Standard Test Method for Measurement of Magnetically Induced Displacement Force," pp. 1-6, 2015.

[7] "ASTM F2213 - 06: Standard Test Method for Measurement of Magnetically Induced Torque on Medical Devices in the Magnetic Resonance Environment 1," pp. 1-8, 2011.

[8] J. F. Schenck, "Safety of strong, static magnetic fields," Journal of Magnetic Resonance Imaging, vol. 12, no. 1, pp. 2-19, 2000.

[9] I. Uwano et al., "Assessment of Sensations Experienced by Subjects during MR Imaging Examination at 7T," Magnetic Resonance in Medical Sciences, vol. 14, no. 1, pp. 35-41, 2015.

[10] F. Schmitt, "The Gradient System." Proc. Intl. Soc. Mag. Res. Med, vol. 21, pp. 1-13, 2013.

[11] B. Yang et al., "Frequency spectrum of the human head-neck to mechanical vibrations," J. Low Freq. Noise, Vib. Act. Control, vol. 37, no. 3, pp. 611-618, sep 2018.

[12] B. Condon and D. M. Hadley, "Potential MR Hazard to Patients With Metallic Heart Valves: The Lenz Effect," Journal of Magnetic Resonance Imaging, vol. 12, no. 1, pp. 171-176, jul 2000.

[13] H. Graf, U. A. Lauer, and F. Schick, "Eddy-current induction in extended metallic parts as a source of considerable torsional moment," Journal of Magnetic Resonance Imaging, vol. 23, no. 4, pp. 585-590, 2006.

[14] L. Golestanirad et al., "Comprehensive analysis of lenz effect on the artificial heart valves during magnetic resonance imaging," Progress In Electromagnetics Research, vol. 128, no. May, pp. 1-17, 2012.

[15] M.-B. Edwards et al., "In vitro assessment of the lenz effect on heart valve prostheses at 1.5 T." J Magn Reson Imaging, vol. 41, no. 1, pp. 74-82, 2015.

[16] F. Shellock, Magnetic Resonance Procedures: Health Effects and Safety, Special Edition. Taylor \& Francis, 2000.

[17] E. M. Maynard, E. Fernandez, and R. A. Normann, "A technique to prevent dural adhesions to chronically implanted microelectrode arrays," Journal of Neuroscience Methods, vol. 97, no. 2, pp. 93-101, apr 2000.

[18] A. Gilletti and J. Muthuswamy, "Brain micromotion around implants in the rodent somatosensory cortex," Journal of Neural Engineering, vol. 3 , no. 3, pp. 189-195, sep 2006.

[19] H. S. Sohal et al., "The sinusoidal probe: a new approach to improve electrode longevity," Frontiers in Neuroengineering, vol. 7, no. April, p. 10, apr 2014 .

[20] W. Zhang, "Mechanical simulation of neural electrode -brain tissue interface under various micromotion conditions," Journal of Medical and Biological Engineering, vol. 34, no. 4, p. 386, 2014.

[21] A. Sridharan, S. D. Rajan, and J. Muthuswamy, "Long-term changes in the material properties of brain tissue at the implant-tissue interface," Journal of Neural Engineering, vol. 10, no. 6, p. 066001, dec 2013.

[22] D. Prodanov and J. Delbeke, "Mechanical and Biological Interactions of Implants with the Brain and Their Impact on Implant Design," Frontiers in Neuroscience, vol. 10, no. February, feb 2016.

[23] E. Fuhrer et al., "Optical gauge head to evaluate gradient field induced vibrations of conductive structures during MRI," in 2017 International Conference on Electromagnetics in Advanced Applications (ICEAA). IEEE, sep 2017, pp. 1555-1558.

[24] A. Lecomte, E. Descamps, and C. Bergaud, "A review on mechanical considerations for chronically-implanted neural probes," Journal of Neural Engineering, pp. 1-33, sep 2017.

[25] R. Pomfret, G. Miranpuri, and K. Sillay, "The Substitute Brain and the Potential of the Gel Model," Annals of Neurosciences, vol. 20, no. 3 , pp. 118-122, 2013.

[26] D. van Dusschoten and M. Wilhelm, "Increased torque transducer sensitivity via oversampling," Rheologica Acta, vol. 40, no. 4, pp. 395399, jul 2001

[27] M. Kavitha and M. R. Reddy, "Characterization of tissue mimicking phantoms for acoustic radiation force impulse imaging," in 2012 IEEE International Conference on Imaging Systems and Techniques Proceedings. IEEE, jul 2012, pp. 553-557.

[28] M. A. Green, L. E. Bilston, and R. Sinkus, "In vivo brain viscoelastic properties measured by magnetic resonance elastography," $N M R$ in Biomedicine, vol. 21, no. 7, pp. 755-764, aug 2008.

[29] S. Budday et al., "Mechanical characterization of human brain tissue," Acta Biomaterialia, vol. 48, pp. 319-340, 2017. 